\title{
ASSEMBLY OF A MICRO BALL-BEARING USING A CAPILLARY GRIPPER AND A MICROCOMPONENT FEEDER
}

\author{
C. Lenders, J.-B. Valsamis, M. Desaedeleer, A. Delchambre, P. Lambert \\ Bio Electro and Mechanical Systems (BEAMS) Department \\ Université Libre de Bruxelles (ULB) \\ Avenue F.D. Roosevelt, 50 CP 165/14 \\ B-1050 Bruxelles, Belgium \\ http://beams.ulb.ac.be
}

\section{Introduction}

Assembly of microsystems is still a challenge today. The numerous parasitic forces often make the manipulation behaviour unpredictable, hence difficult to automate. Therefore special designs of grippers and adequate strategies have to be implemented. Recent works have shown that capillary forces are strong enough to be used to manipulate components in microassembly technology [1]. However, many investigations need to be performed concerning the manufacturing of a microgripper for an industrial use. If the feasibility has been theoretically shown, there is still work to be achieved over the practical implementation of such a gripper.

The purpose of this paper is to present an assembly station for a watch bearing including a component feeder: the balls delivered in bulk are separated and arranged in specific holes. Then a gripper picks five balls successively and places them in the housing of the bearing.

The station we present here uses the capillary gripper designed by Lambert [2]. This gripper is integrated on a precision $x y z$ positioning table. Since electrostatic repulsion and capillary adhesion are still an issue with this kind of gripper during the gripping step, a microcomponent feeding device has been implemented to supply the gripper.

This paper falls into 5 parts: Section 2 describes the problem while Section 3 focuses on the design of the solution, concerning the feeder and the gripper. We present the experimental setup in Section 4, and the results in Section 5. Finally, we give conclusions in Section 6. 


\section{Problem definition}

The problem can be divided into several independent sub-problems. A first set of problems is linked to the balls feeding. A second one is linked to the micromanipulation of the balls (picking, moving and placing).

\subsection{Microcomponent feeding system}

Because of the scaling effects, many parasitic forces are becoming predominant at microscale, which may disrupt the gripping process. The two major actors are the electrostatic forces and the capillary forces generated by liquid bridges between solids.

The electrostatic forces arise mainly from the unpredictable triboelectrification and may either repulse the component from the gripper, or attract it. In the case of repulsion, the gripper may never reach the component. This problem is illustrated in Fig. 1(a).

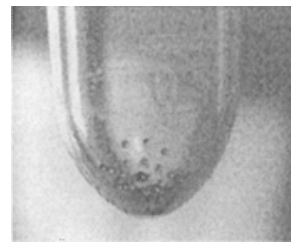

(a) Illustration of electrostatic repulsion: the ruby balls $(\varnothing 0.5 \mathrm{~mm})$ repel each other due to triboelectrification

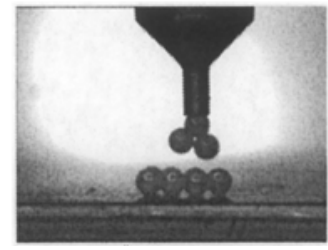

(b) The capiliary forces due to liquid bridges may be large enough to stick the components

(alumina ball, $(\varnothing 0.5 \mathrm{~mm})$ together

Fig. 1. Example of repulsive and attractive parasitic forces

The capillary forces are indeed present in this kind of problem either by putting liquid for the purpose of surface tension gripping [1] or because of the relative humidity [3]. In some cases, the force may be large enough to stick different components together, as illustrated in Fig. 1(b). It is then difficult to grip them one by one.

The solution proposed to minimise these parasitic effects is to work in an immersed environment, as already proposed by the Automation and Micromechatronics Department, FEMTO-ST Institute, Besançon, France. The design shall comply with the following specifications (illustrated in Fig. 2):

- The components are supplied in bulk

- The components are supposed to be spherical, with a diameter of $500 \mu \mathrm{m}$

- The final location of the components must be known

- The capillary gripper should be able to catch one component

- When the capillary gripper catches a component, it must grip only one 


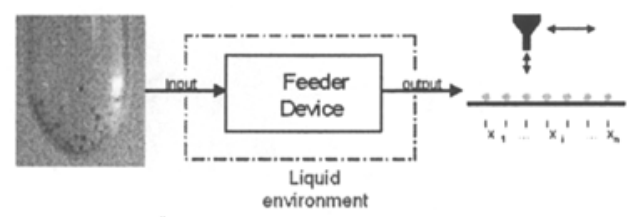

Fig. 2. Illustration of the specifications for the microcomponent feeding system

\subsection{Microassembly system}

Once the balls are arranged, the bearing is assembled with a capillary gripper. The gripper is dived in a liquid bath to form a liquid drop on its tip. Then the gripper picks one ball at a time, places it in an empty hole on the ball bearing and releases it. Problems appearing during the manipulation are described below.

- The picking phase consists in taking the ball out of its hole. The main problems are the checking of the presence of a ball in a feeder, the strength of the capillary forces which should be large enough to extract the ball.

- The placing phase is the positioning of the ball into a determined hole of the ball bearing. The precision of placing should involve a control of the relative position of the ball with regard to the gripper and a good precision of the $x y$-axes.

- The releasing phase consists in breaking the ball away. Several strategies exist: deforming the liquid bridge (changing the curvature [4], by using external hydrophobic materials), using external forces (pulling forces such as sticking or soldering the component, and shearing forces).

The next step is now to repeat the sequence five times in order to fill each housing of the bearing.

\section{Solutions design}

\subsection{Feeding device}

As announced in the previous section, the cell of the feeding device will be filled with liquid to avoid parasitic electrostatic and capillary forces. Since there is no free surface, capillary forces are totally cancelled in immersed environment, while the permittivity of water, which is 80 times larger than that of dry air, reduces the electrostatic force drastically. Moreover, if the liquid is a conductive media, the electrostatic charges accumulated on the components could disappear. Of course, because we use a capillary gripper to catch the components, we have to extract the liquid from the cell before the picking step.

In order to fulfil the requirements, we propose to create a housing for each component. This housing will separate the balls and maintain them on a specific loca- 
tion. It will also allow the gripper to catch the component. Hence the housing shall be large enough to allow the gripper to pass. The conformity with the ball must also be as low as possible, meaning that the residues of liquid should not develop a capillary force larger that the gripping force (see Fig. 3).

The geometry chosen (Fig. 4(a)) is a thin metal plate (1), the mesh, with in-line holes (3). To prevent the ball from passing through and yet to allow the liquid to flow in order to empty the cell, we have placed a vertical plate (2) beneath the holes. This also gives us a low conformity.

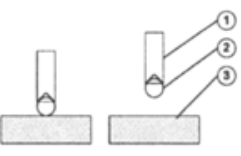

(a)

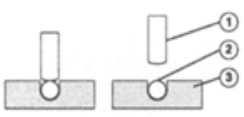

(b)

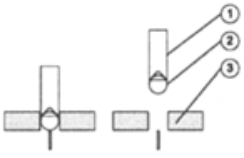

(c)

Fig. 3. Illustration of the conformity issue. If the shape of the component matches better the gripper than the housing (a), the capillary force generated by the liquid bridge between the component and the gripper is larger than that of the liquid bridge between the component and the housing. Otherwise, the gripper shall not be able to extract the component (b). With our design (c), the conformity is kept low thanks to the thin vertical plate beneath the housing.

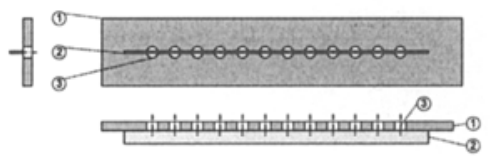

(a) Schematic view of the ball feeder
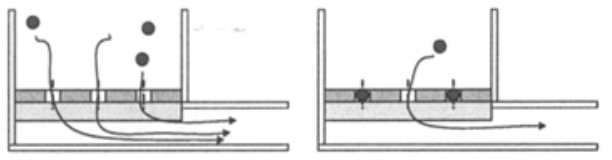

(b) Schematic view of the balls flow. When a housing is filled with a component, the streamline is cut and the remaining components are guided to the free locations

Fig. 4. Design and working of the feeder

\subsubsection{Perspective}

So far, the cell is tilted manually in order to move the components toward the housings. We intend to improve the device by using the hydrodynamic forces in this liquid. By flowing the fluid to the housings, we will guide the components to these locations. To achieve this, we have placed a channel below the mesh to allow the fluid to flow. It is also used to empty the cell when the components have reached their location.

The advantage of this design is that when a location is filled with a component, no more streamline passes through this hole, hence guiding the remaining components to the other free locations (see Fig. 4(b)).

To generate this streamline, we shall use a pumping device ((IV) on Fig. 6) to create a velocity and henceforth a depression in the lower channel, and inject the fluid back in the cell (III) through a buffer (I). Finally, a valve (II) will be used to 
control the flow between the buffer and the cell. A complete sequence is described schematically below (Fig. 6).

\subsection{Modelling}

This principle has been checked by simulation on Comsol Multiphysics software (see Fig. 5).

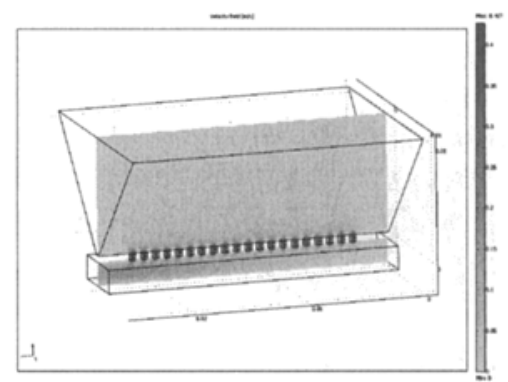

(a) 3D simulation of the flow in the feeder. With the particle tracing option, we have an information about the behaviour of the ball in the cell.

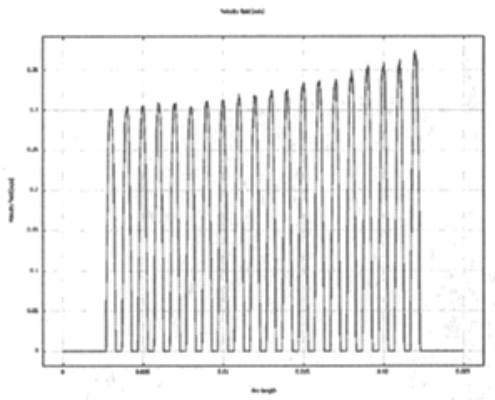

(b) Evolution of the velocity of the fluid above the different housing of the mesh (outlet is under the right-hand side)

Fig. 5. Flow simulation using Comsol Multiphysics. With this simulation, we can see what are the paths of the streamlines. This should help us to estimate the best position to inject the components

We have approached the geometry of the feeder cell, and computed NavierStokes equation to find the pressure and velocity field. In this simulation, we have supposed that the liquid feedback was injected uniformly on the free surface.

The particle tracing option of Comsol Multiphysics makes an estimation of the movement of particles in the fluid (the flow is assumed not to be modified by the particle movement). This will help us to optimise the parameters and the geometry of the device.

Since we are pumping the fluid on one side of the cell, the flow will not be uniform in all the housings. This simulation allows us to check if the flow is not too low on the opposite side of the mesh. 

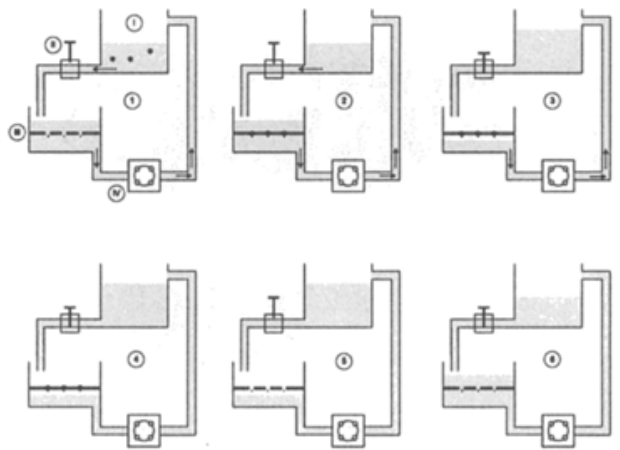

Fig. 6. General diagram of the whole component feeder. The components are unloaded in the buffer (1). They are directed to the housing in the cell (2). Then the valve is closed to eliminate the liquid surrounding the components (3). The gripper picks up the components (4), and then the valve is opened (5) to refill the cell (6).

\subsection{Microassembly system}

The gripper (design taken from [1]) is shown in Fig. 7. It is made of a stainless steel cylindrical tip with a conical concavity, in order to improve the conformity between the gripper and the ball. The cylinder diameter agrees with the ball diameter which is $0.5 \mathrm{~mm}$.

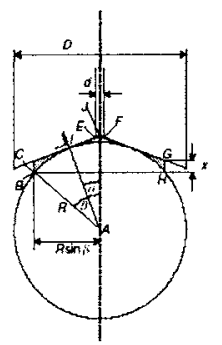

Fig. 7. Gripper design, from [1]. Main parameters are the diameter $D$, the angle spread $\pi-2 \alpha$

Capillary forces depend in a first approximation on the surface contact. In our design, cohesive forces of the ball are higher with the gripper than the feeder. Indeed, if the ball is centred on the gripper, the contact line is $\pi D \cos (\alpha)$ while the contact between the feeder and the ball is 2 points as shown in Fig. 8(a). Fig. 8(b) shows the evolution of the capillary forces on successive picking of a ball [5] with only one feeding in liquid. 


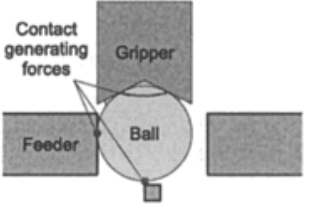

(a) Contact zone were capillary forces will appear

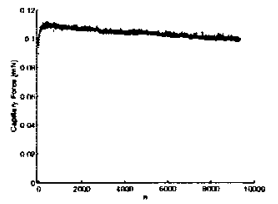

(b) Capillary forces generated by the gripper

Fig. 8. Capillary forces generated with our gripper during almost 10000 picking

During the placing phase, the main advantage of our gripper design is the autocentring of the ball, avoiding having to control the ball position with regard to the gripper (Fig. 9(a)). Furthermore the ball can roll in the destination hole (Fig. 9(b)). Theoretically the accuracy should be below the size of the radius that is widely achieved (see Sect. 4). As a result, we will study the link between the initial moving off centre $(\Delta x)$ on the residual moving off centre $(\delta x)$ and the maximum tolerance on the placement accuracy $(\Delta u)$.

Finally the last stage consists on releasing the ball. In this paper, we will use the cage as a stop that will produce a shearing on the liquid bridge (Fig. 9(c)).

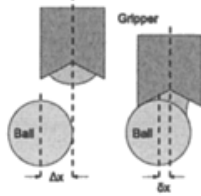

(a) Centering error

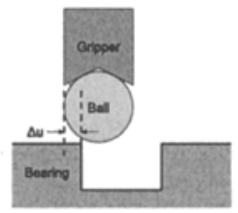

(b) Positioning error

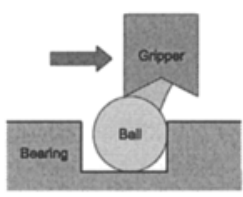

(c) Releasing strategy

Fig. 9. Concept in placing and releasing phases

\section{Experimental setup}

The manipulation is mounted on a station with three degrees of freedom (one per translation axis). The station is made of a gripper, a component feeder and a ball bearing placed on a support.

Horizontal axes are decoupled from the vertical one according to the split-axes concept [6]: the gripper has the vertical motion (z-axis). The feeder and the bearing are mounted on a table equipped with the two horizontal motions ( $x y$-axes).

The vertical motion is controlled by a linear motor (a SMAC linear actuator LAL95-050-71 and a LAC-1 controller, accuracy $1 \mu \mathrm{m})$. The motion of $x y$-table is controlled by two brushless DC servo-motor (SMART SM2315D, from Animatics, accuracy $20 \mu \mathrm{m}$ ). Rotative motion is converted into a linear motion thanks to linear guides with a worm screw (THK, KR2001A). Two optical devices (a Keyence laser 
displacement sensor LC-2440, accuracy $0.2 \mu \mathrm{m}$ and a controller unit LC-2400W) allow us to get the position of the table according to the gripper.

\section{Results}

\subsection{Feeding device}

So far, the feeder has been tested manually. The components were injected in still water, on one end of the mesh, and the device was tilted until the components reached the other side. A small pool was placed next to the cell to catch the balls that did not fall into a housing. Fig. 10 shows the feeder used manually. With the manual feeder, we have measured the filling ratio (number of filled housing on total number of housing). The results are shown in Table 1.

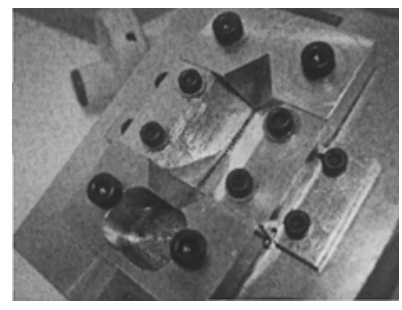

Fig. 10. Picture of the feeder with the recovery pool. The components are injected on the other end. The device is tilted and the components slide towards the recovery pool

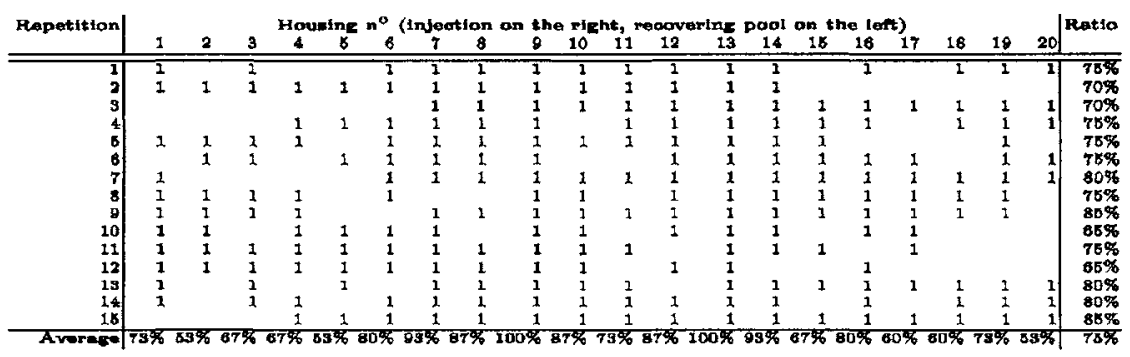

Table 1. Filling ratio measurement on the manual device. The average filling ratio is $75 \%$

We have also shown that it was possible to extract the balls from the mesh (Fig. 11). It is however necessary to wait several minutes for the liquid remaining in the housing to evaporate. If the liquid is water, the time before picking the balls is larger than 10 minutes. To reduce it, we have used a solution of ethanol and ether. 
In this case, the time to wait before picking the components is reduced down to 5 to 10 minutes. To reduce this delay, we propose to blow heated air above the mesh, or to heat the mesh itself by flowing an electrical current. Otherwise, the influence of the delay on the entire assembly station could be reduced by parallelizing several feeders.

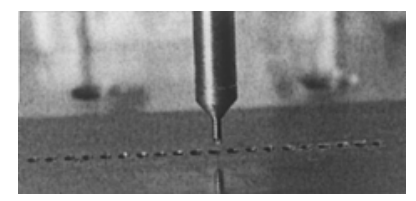

Fig. 11. Extraction of a ball out of the mesh with the capillary gripper

With the automation proposed in Section 3.1, we still have to optimise the system. The main parameters are the viscosity and density of the fluid, the velocity of the fluid and the geometry of the device. We shall estimate the influence of these parameters on the filling ratio (i.e. how many housing are filled with a component) and the cycle time, which is mainly due to the time to wait for the residues of liquid to evaporate. This will allow us to find the best combination to enhance the performances of the device.

Concerning the tolerance of the positioning for the picking phase, we have observed that the auto-centring is only working if $\Delta x$ is below $31 \mu \mathrm{m}$ (measured in the worst case, i.e. with minimum amount of liquid). This value allows us to estimate the positioning accuracy needed during the picking phase.

The maximal tolerance on the placement accuracy for the release task is mainly due to the geometry of the gripper and the final location (see Fig. 12). The calculation gives a result of $\Delta u=164 \mu \mathrm{m}$, while we experimentally encounter problems to insert the ball in the bearing if $\Delta u \geq 125 \mu \mathrm{m}$.

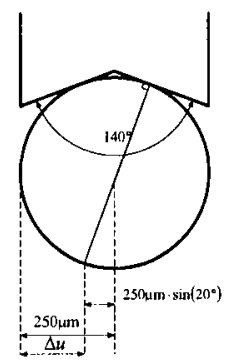

Fig. 12. Positioning tolerance in releasing task is defined by the geometry of the system

We also measured the cycle time of picking, placing and releasing steps on our experimental setup. Typical values are $2.56 \mathrm{~s}$ for picking a component, $0.14 \mathrm{~s}$ to release it, and a total of $6.15 \mathrm{~s}$ to wet the gripper tip in a water tank and to move the gripper to the different locations (component feeder, assembly area and water tank). 
The picking phase is slow in order not to create a shock on the system, but the placing phase is constrained mainly by the actuators speed.

\section{Conclusion and future work}

In this paper, we have designed a manipulation station from the balls in bulk to their assembly in a ball bearing. We try to characterise the feeder, the gripper and the station by showing the success rate of the manipulation, the cycle time and the tolerance on the accuracy.

As future work, we could continue the automation of the station. The feeder could work automatically with different pumps and a system that drain the excess of balls. A vision system could be attached to watch if holes are provided with balls (since the feeding ratio is below 100\%) and the number of balls leaving in order to restart the feeder. Vision system could equally check the presence of a ball on the gripper and presence in the bearing.

Another investigation is to study the scale effect by using a smaller ball with a diameter of $300 \mu \mathrm{m}$.

\section{Acknowledgements}

J.-B. Valsamis is a researcher funded by a grant of the F.R.I.A. - Fonds pour la Formation à la Recherche dans l'Industrie et l'Agriculture.

\section{References}

1. P. Lambert, F. Seigneur, S. Koelemeijer, and J. Jacot, "A case study of surface tension gripping: the watch bearing," J. Micromech. Microeng., vol. 16, no. 7, pp. 1267-1276, 2006.

2. P. Lambert, "A contribution to microassembly: a study of capillary forces as a gripping principle," Ph.D. dissertation, Université libre de Bruxelles, Belgium, 2004.

3. A. Chau, S. Régnier, A. Delchambre, and P. Lambert, "Three dimensional model for capillary nanobridges an capillary forces," Model. Simul. Mater. Sci. Eng., vol. 15, pp. 305 $317,2007$.

4. F. Biganzoli, I. Fassi, and C. Pagano, "Development of a gripping system based on capillary force," in Proceedings of ISATP05, Montreal, Canada, 19-21 July 2005, pp. 36-40.

5. J.-B. Valsamis, A. Delchambre, and P. Lambert, "An experimental study of prehension parameters during manipulation task," in Proc. of the 5th International Workshop on Microfactories, 25-27 October 2006.

6. S. van Gastel, M. Nikeschina, and R. Petit, Fundamentals of SMD Assembly. Assembléon Netherlands B.V., 2004. 